\title{
Internet-based interventions for eating disorders in adults: a systematic review
}

Ruth Dölemeyer ${ }^{1,2^{*}}$, Annemarie Tietjen ${ }^{1}$, Anette Kersting ${ }^{1,2}$ and Birgit Wagner ${ }^{1,2}$

\begin{abstract}
Background: This systematic review evaluates the efficacy of internet-based interventions for the treatment of different eating disorders in adults.

Method: A search for peer reviewed journal articles detailing Randomised Control Trials (RCT) and Controlled Trials (CT) addressing participants with eating disorders aged at least 16 was completed in the electronic databases Web of Science, Psyclnfo and PubMed. The quality of the included articles was assessed, results were reviewed and effect sizes and corresponding confidence intervals were calculated.

Results: Eight studies, including a total of $\mathrm{N}=609$ participants, fulfilled the selection criteria and were included. The majority of treatments applied in these studies were based on CBT principles. Six studies described guided selfhelp interventions that showed significant symptom reduction in terms of primary and secondary outcomes regarding eating behaviour and abstinence rates. These studies produced significant medium to high effect sizes both within and between the groups after utilisation of guided self-help programs or a self-help book backed up with supportive e-mails. The two remaining studies utilised a specific writing task or e-mail therapy that did not follow a structured treatment program. Here, no significant effects could be found. Treatment dropout rates ranged from $9 \%$ to $47.2 \%$. Furthermore, reductions in other symptoms, for example depression and anxiety, and an increase in quality of life were found by four studies.
\end{abstract}

Conclusions: Overall, the results support the value of internet-based interventions that use guided self-help to tackle eating disorders, but further research is needed due to the heterogeneity of the studies.

Keywords: Eating disorders, Internet-based intervention, Systematic review, Binge eating disorder, Bulimia nervosa, EDNOS

\section{Background}

Eating disorders are associated with both high social and personal costs for the person concerned. Most people with eating disorders do not access effective treatment [1] and show a preference for low-threshold interventions rather than conventional health care provided for mental health problems $[2,3]$. For this reason, an increasing number of internet-based interventions addressing eating disorders have been developed to facilitate access to effective treatments for these individuals. Many of these internet-based programs have been developed with the aim of preventing eating disorders [3-11], but more recently

\footnotetext{
* Correspondence: ruth.doelemeyer@medizin.uni-leipzig.de

'Department of Psychosomatic Medicine and Psychotherapy, University of Leipzig, Semmelweisstr. 10, Leipzig 04103, Germany

${ }^{2}$ Leipzig University Medical Center, IFB AdiposityDiseases, Leipzig, Germany
}

there has also been an increase in interest in internetbased interventions targeting people who already suffer from a diagnosed eating disorder [12-19]. While internetbased interventions addressing bulimia nervosa [14-19], binge eating $[12,13,16]$, EDNOS (eating disorder not otherwise specified) $[17,19]$ and body dissatisfaction $[20,21]$ have been conducted, there is still a relatively low number of investigations into the application of internet-based interventions for anorexia nervosa. It can be assumed that this is due to the fact that the weight loss accompanying anorexia nervosa can be life threatening, making it the eating disorder with the highest mortality rate [22].

In general, internet-based interventions have several advantages, for example the lack of geographic boundaries, enabling widespread dissemination of treatment [23]. Furthermore, internet-based interventions are cost-effective

\section{Biomed Central}


[24] and provide greater user control, flexibility, open access and anonymity [25]. They are therefore especially relevant for patients who might not otherwise access treatment for reasons such as fear of social stigma or lack of easy access to a treatment centre.

While a number of reviews and meta-analyses of internet-based interventions have been published, e.g. for depression [26,27], depression and anxiety [28-31], obesity [32] and the prevention of eating disorders [3], there is, to our knowledge, no review examining the efficacy of internet-based interventions for the treatment of existing eating disorders. Thus, with this systematic review we aim to give an overview of the different forms of internet-based interventions that have been applied for people suffering from an eating disorder.

The following key questions are addressed by this systematic review:

1. What is the evidence for the value of internet-based interventions for the treatment of eating disorders? To answer this question, effect sizes and corresponding confidence intervals were calculated for within and between group analyses. Additionally, rates of abstinence and dropout were taken into account.

2. What factors are associated with these treatment effects (e.g. duration of treatment, degree of therapist involvement)?

To answer these questions only analyses of quantitative data were taken into account; qualitative data were not considered at this time.

\section{Methods}

Before starting with literature search for the systematic review, inclusion criteria and methods of analysis were specified. These criteria have not been documented in an official review protocol.

\section{Study eligibility}

Studies were selected and included in the present review according to five criteria: (1) publication in a peer reviewed journal, (2) presence of a controlled design, (3) inclusion of the internet as at least one mode of delivery for treatment or self-help, (4) inclusion of participants aged at least 16 years and suffering from an eating disorder and (5) presence of changed eating behaviour as a primary outcome. Studies were excluded if they (1) addressed prevention of eating disorders, (2) addressed weight loss programs, or (3) did not include subjects suffering from a diagnosed eating disorder.

\section{Study selection}

For the selection of studies, Web of Science, PsycInfo and PubMed were electronically searched for articles published or e-published before November 2012 by combining the terms "random" the terms "eating disorder", "anorexia", "bulimia", "binge eating" OR "EDNOS", the terms "online", "internet", "computer", "email" OR "web" and the terms "intervention", "therapy" "self-help", "treatment" OR "program" in titles or abstracts. No limitation was made regarding the language of articles. The titles and abstracts of the 460 articles identified by the initial search were screened to determine their relevance to the review. Articles that did not meet inclusion criteria were excluded at this stage, whereas the full text of potentially relevant studies was examined. Furthermore, the reference lists from retrieved articles were checked for additional relevant literature. The selection of articles was independently performed by the primary and secondary author of this review.

\section{Data extraction}

Data extraction was conducted independently by two authors, consulting a third reviewer in the case of discrepancy in the documentation of study features. Variables extracted included the authors of the study, title and publication year, information regarding number, diagnosis, gender and age of participants, characteristics of intervention and control groups (e.g. frequency of contact, kind of control group), duration of treatments, time points of assessment, and measures used for outcome assessment. The rates of study dropouts (regarding percent of missing post-treatment assessments over all groups) and treatment dropouts (regarding percent of participants not finishing treatment in the intervention group) were also noted, as were rates of abstinence. Furthermore, information necessary for evaluating methodological quality was extracted.

\section{Assessment of methodological quality}

Methodological quality was assessed using an 11-item list oriented on a scale developed by van den Berg et al. [33]. Studies were rated independently by the first author and checked by the second author. Disagreements were discussed until consensus was reached. Each item was rated as yes, no, or unknown. A total methodological quality score (ranging from $0-11$ ) was calculated by summing up all yes items. Studies were rated as having good methodological quality if they met at least twothirds of the criteria (eight or more items).

\section{Assessing the effects of internet-based interventions for eating disorders}

To answer the key questions of this review, key eating disorder-related symptoms (e.g. bingeing and purging) and rates of abstinence were considered as primary outcomes. As secondary outcomes, results of key questionnaires were considered. Included were questionnaires 
that are commonly used for the assessment of symptoms related to eating disorders, resulting in examination of (1) EDE interview and the Eating Disorder Examination Questionnaire (EDE-Q) [34], a semi-structured interview and its self-report version, measuring the core psychopathology of eating disorders; and (2) the Eating Disorder Inventory (EDI) and Eating Disorder Inventory 2 (EDI-2) [35-38], which were developed to assess psychological characteristics of patients with eating disorders. In studies that did not include one of these questionnaires, (3) the Bulimia Investigatory Test Edinburgh (BITE) [39], a self-rating measure assessing symptoms of $\mathrm{BN}$, was considered.

If a study measured outcomes across several time points, the first time point after completion of the intervention was selected for comparison of studies. For studies that included more than one control group, the one with the least contact was selected for comparison. Since statistical significance of t-tests depends upon sample size, level of significance, tests used and other variables of study design, effect sizes (ES, Hedges' g) were calculated by the authors, according to Hedges [40]. For effect size calculation, intention to treat (ITT) data was used. Where ITT data were not available, ITT effects were estimated, assuming a zero effect for study dropouts. If no data for calculating or estimating effect sizes were available, results of ANOVAs or effect sizes reported in the studies were used. An ES of less than 0.5 was interpreted as small, 0.5 to 0.8 as medium and greater than 0.8 as large [41]. Additionally, 95\% confidence intervals (CIs) were calculated according to Hedges and Olkin [40]. Furthermore, where follow-up data were available, stability of the effects was reported and additional outcomes (e.g. depression, anxiety or quality of life) were considered. Here, the (1) Beck Depression Inventory (BDI) [42], or BDI-II [43], (2) the Hospital Anxiety and Depression Scale (HADS) [44,45] or (3) the Montgomery Åsberg Depression Scale Self-assessment (MADRS) [46] were used to assess symptoms of depression and anxiety. To assess quality of life, (1) the short form of the Impact of Weight on Quality of Life (IWQOL-Lite) [47], (2) the Satisfaction with Life Scale (SWLS) [48] and (3) the WHO Quality of Life Questionnaire (WHOQOL-Bref) [49] were included in the review.

Because the studies differ in terms of the eating disorders addressed, characteristics of participants and internet-based programs applied, no meta-analysis was performed but results will be presented for each study and conclusion will be drawn.

\section{Results}

Study selection

The search and selection process for articles is illustrated in Figure 1. A total of 651 articles were identified by the initial search. After removing duplicate articles $(\mathrm{n}=191)$ and irrelevant studies $(n=436), 24$ articles were retained for further consideration. Of these, 16 articles were excluded as they addressed prevention of eating disorders or presented data from participants who did not fulfil a diagnosis of an eating disorder $(\mathrm{n}=6)$, they did not use a controlled design $(n=5)$, they were addressing relapse prevention $(n=1)$ or they did not use internet-based therapy as the mode of delivery $(n=4)$. Screening the reference lists from retrieved articles did not lead to the inclusion of any additional relevant literature $\rightarrow$ Figure 1 .

\section{Methodological quality}

In Table 1 the results of the methodological quality assessment are described. Of the eight studies included, five [12,16-19] were rated as having good methodological quality, whereas two studies $[13,15]$ just missed this rating by meeting seven rather than eight out of the 11 criteria. One study [14] failed to report the eligible criteria and timing of outcome measurements between groups were not comparable.

\section{Methods}

Tables 2 and 3 provide an overview of the methods used in the studies, detailing the intervention, control group and participant characteristics as well as the diagnosis addressed by each study. Furthermore, the time points of assessments are listed and rates of abstinence and dropout are reported. The eight included studies all were published in English and described different interventions using the internet as a mode for delivery of treatment or self-help support. Three of the studies focused on bulimia nervosa $[14,15,18]$, two on full or subthreshold criteria for binge eating disorder [12,13], while the remaining three studies addressed more than one kind of eating disorder $[16,17,19]$. Eating disorders were diagnosed according to DSM-IV criteria in all but two studies $[15,18]$. While six of the studies were randomised controlled trials [12,15-19], two studies were controlled but not randomised $[13,14]$. Of the studies included in this review, two did not assess the stability of effects $[14,17]$ while in the remaining studies the time frame for follow-up ranged between 8 weeks [15] and 12 months after end of the treatment [18].

\section{Participants' characteristics}

The included studies involved a total of $\mathrm{N}=609$ participants suffering from serious eating issues or diagnosed eating disorders, with sample sizes ranging from 42 [13] to 97 [17]. About $97 \%$ of the total sample was female. Five of the studies included both genders [15-19] while three only addressed women [12-14]. The average age of participants ranged between 23.7 and 44.6 years. 


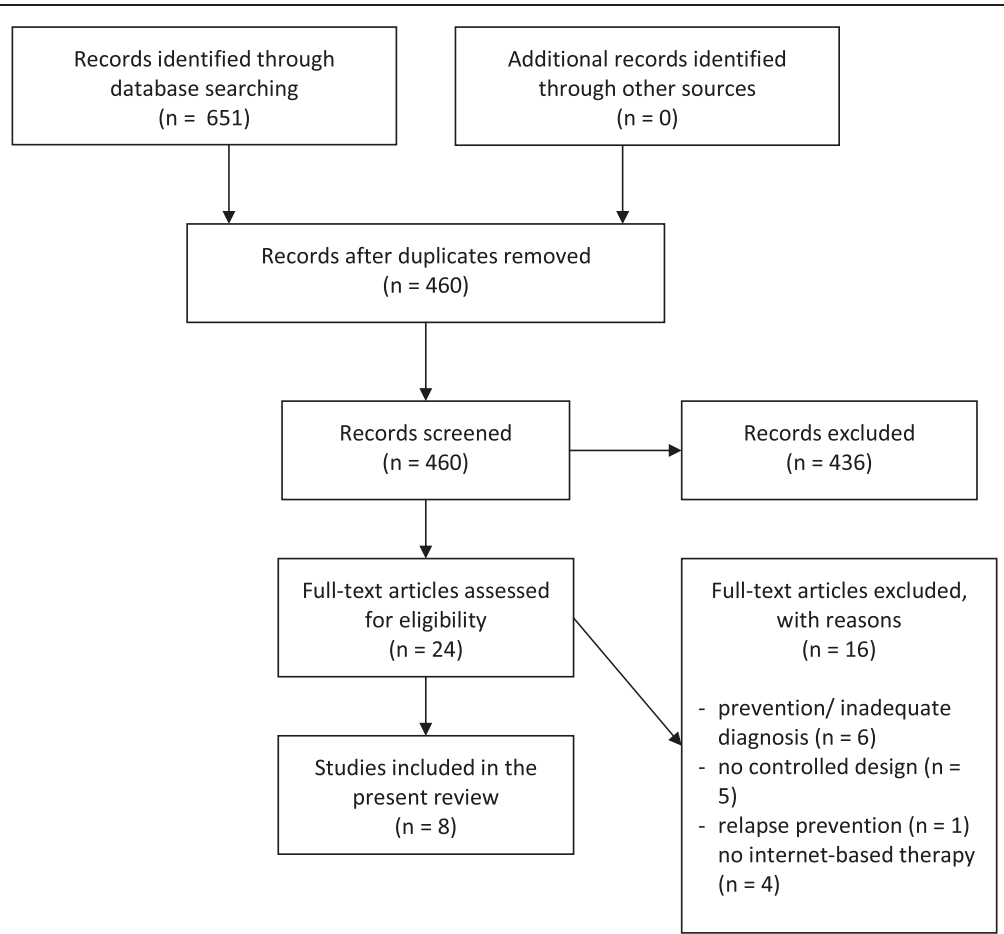

Figure 1 Identifying studies for inclusion in systematic review.

\section{Intervention characteristics}

Cognitive behavioural therapy (CBT) or modules of CBT formed the basis of all but one intervention [15]. This exception involved an expressive writing task that, according to Pennebaker [50], incorporated exploration of the patients' thoughts and emotions. Most of the approaches additionally implemented psychoeducational elements. Treatment programs differed in length, ranging from three days for the shortest [15] up to six months for the two longest $[12,13]$. In brief, six studies offered the patients a guided self-help intervention [12-14,16,18,19], one utilised a specific writing task [15] and one used e-mail therapy that did not follow a structured treatment program [17]. Five of the six studies that used guided self-help interventions developed and used structured treatment programs [12-14,18,19], while in one study [16] the self-help intervention utilised a book with accompanying tasks and homework. The extent of therapist support or guidance in the various internetbased interventions differed from no support at all [15] to an average of two emails per week over the course of the treatment period [17]. In most studies, an average of one contact per week between coaches and participants was planned.

\section{Control group characteristics}

Four studies utilised a comparison group that was a waiting list control group with no professional contact at all [13,17-19]. In three studies, waiting list control groups received exercises to do while waiting $[12,14,16]$ and two of the studies included an additional control group $[17,18]$. Finally, the study using the expressive writing task as intervention program [15] did not use a waiting list control group at all, but advised participants in the control group to write about superficial topics, in a factual manner, without exploring thoughts or feelings.

\section{Outcomes}

\section{Outcomes related to eating disorders}

Table 4 illustrates the effect sizes for primary and secondary outcomes, as well as the corresponding CIs. In two studies $[12,18]$, means and standard deviations for the calculation of effect sizes were available in the form of ITT data, while four studies $[13,15,16,19]$ reported these in terms of completers' data. The remaining two studies did not provide the information required for calculating effect sizes. For one study [14], no standard deviations or test-values were given. Here effect sizes for between groups were taken from reported study results and reported confidence intervals for mean differences were considered. For the other study that did not provide standard deviations [17], F-values were reported but estimation of effect sizes were not possible as the study design included three groups. Here, results 
Table 1 Assessment of methodological quality

\begin{tabular}{|c|c|c|c|c|c|c|c|c|}
\hline Items & $\begin{array}{l}\text { Ljotsson } \\
\text { et al. [16] }\end{array}$ & $\begin{array}{l}\text { Robinson \& } \\
\text { Serfaty [17] }\end{array}$ & $\begin{array}{l}\text { Johnston } \\
\text { et al. [15] }\end{array}$ & $\begin{array}{l}\text { Carrard } \\
\text { et al. [12] }\end{array}$ & $\begin{array}{l}\text { Sanchez-Ortiz } \\
\text { et al. [19] }\end{array}$ & $\begin{array}{l}\text { Fernandez-Aranda } \\
\text { et al. [14] }\end{array}$ & $\begin{array}{l}\text { Carrard } \\
\text { et al. [13] }\end{array}$ & $\begin{array}{l}\text { Ruwaard } \\
\text { et al. [18] }\end{array}$ \\
\hline \multicolumn{9}{|l|}{ Methodological quality } \\
\hline $\begin{array}{l}\text { Were the eligible } \\
\text { criteria specified? }\end{array}$ & yes & yes & yes & yes & yes & no & yes & yes \\
\hline $\begin{array}{l}\text { Was the method of } \\
\text { randomization } \\
\text { described? }\end{array}$ & yes & yes & no & yes & yes & yes & yes & yes \\
\hline $\begin{array}{l}\text { Were the groups } \\
\text { similar at baseline } \\
\text { regarding important } \\
\text { prognostic indicators? }\end{array}$ & yes & yes & unknown & yes & yes & yes & yes & yes \\
\hline $\begin{array}{l}\text { Were the index and } \\
\text { the control interventions } \\
\text { explicitly described? }\end{array}$ & yes & yes & yes & yes & yes & yes & yes & yes \\
\hline $\begin{array}{l}\text { Was the outcome } \\
\text { assessor blinded to } \\
\text { the interventions? }\end{array}$ & Unknown & yes & Unknown & no & yes & Unknown & no & Unknown \\
\hline $\begin{array}{l}\text { Was the dropout rate } \\
\text { described and were the } \\
\text { characteristics of } \\
\text { dropouts compared } \\
\text { with the completers? }\end{array}$ & yes & yes & yes & yes & yes & yes & $\begin{array}{l}\text { No (no } \\
\text { comparison) }\end{array}$ & no \\
\hline $\begin{array}{l}\text { Was long-term follow- } \\
\text { up in the groups } \\
\text { comparable? }\end{array}$ & no & no & yes & yes & no & no & yes & yes \\
\hline $\begin{array}{l}\text { Was the timing of the } \\
\text { outcome measurements } \\
\text { in the groups comparable? }\end{array}$ & yes & yes & yes & yes & yes & no & yes & yes \\
\hline $\begin{array}{l}\text { Was the sample size of } \\
\text { each group described } \\
\text { by means of a power } \\
\text { calculation? }\end{array}$ & no & yes & yes & yes & yes & no & no & yes \\
\hline $\begin{array}{l}\text { Did the analysis include } \\
\text { intention-to-treat analysis? }\end{array}$ & yes & yes & no & yes & yes & Unknown & no & yes \\
\hline $\begin{array}{l}\text { Were point estimates and } \\
\text { measures of variability } \\
\text { presented for the primary } \\
\text { outcome measures? }\end{array}$ & yes & $\begin{array}{l}\text { Mean but no } \\
\text { standard deviation }\end{array}$ & yes & yes & yes & $\begin{array}{l}\text { Mean but no } \\
\text { standard deviation }\end{array}$ & yes & yes \\
\hline
\end{tabular}

of analyses of variance reported in the study were considered instead.

\section{Primary outcomes}

Figure 2 shows effect sizes and corresponding confidence intervals for bingeing and purging. Six of the studies assessed bingeing or purging, while in two studies $[15,17]$ these behaviours were not assessed. Medium to large effect sizes from pre- to post-treatment were found in the intervention group for both bingeing and purging episodes, ranging from 0.75 to 1.05 for binge episodes and from 0.41 to 0.77 for purging. When effect sizes were calculated between groups, however, a significant reduction in the number of binge episodes in the intervention group as compared to the control group was only found in two studies [12,16], with moderate effect sizes. In studies that assessed purging behaviour, no significant differences in reduction of purging between groups were found, with one exception. One study [16] found a significant reduction in purging behaviour in the intervention group compared to the control group, with a considerably high effect size of 1.63. Sanchez-Ortiz and colleagues [19] additionally assessed frequency of vomiting and found medium-sized effects within the treatment group, while effects were only small when the two groups were compared. In contrast to this, FernandezAranda et al. [14] assessed frequency of vomiting and reported high effect sizes after comparing the two groups.

The definition of abstinence differed between studies and the rates of abstinence in the intervention groups were found to vary widely in the different studies between $22.6 \%$ [14] and 45\% [13]. While in most studies, abstinence was defined as absence of the relevant eating disorder behaviour (e.g. bingeing or purging) over a 
Table 2 Characteristics of treatment programs applied in included studies

\begin{tabular}{|c|c|c|c|c|c|c|c|c|}
\hline Authors & $\begin{array}{l}\text { Ljotsson } \\
\text { et al. [16] }\end{array}$ & $\begin{array}{l}\text { Robinson \& } \\
\text { Serfaty [17] }\end{array}$ & $\begin{array}{l}\text { Johnston } \\
\text { et al. [15] }\end{array}$ & Carrard et al. [12] & $\begin{array}{l}\text { Sanchez-Ortiz } \\
\text { et al. [19] }\end{array}$ & $\begin{array}{c}\text { Fernandez- } \\
\text { Aranda et al. [14] }\end{array}$ & Carrard et al. [13] & Ruwaard et al. [18] \\
\hline $\begin{array}{l}\text { Type of study/ } \\
\text { Randomisation }\end{array}$ & $\begin{array}{l}\text { RCT: based on } \\
\text { generated random } \\
\text { numbers; Stratification } \\
\text { procedure was } \\
\text { implemented with } \\
\text { regard to diagnosis } \\
\text { and severity }\end{array}$ & $\begin{array}{l}\text { RCT: randomization } \\
\text { was based on } \\
\text { generated random } \\
\text { numbers }\end{array}$ & $\begin{array}{l}\text { RCT: No exact } \\
\text { description was } \\
\text { given }\end{array}$ & $\begin{array}{c}\text { RCT: Randomization } \\
\text { was based on } \\
\text { generated random } \\
\text { numbers }\end{array}$ & $\begin{array}{c}\text { RCT: based on } \\
\text { generated random } \\
\text { numbers; } \\
\text { Stratification } \\
\text { procedure was } \\
\text { implemented with } \\
\text { regard to diagnosis } \\
\text { and recruitment site }\end{array}$ & $\begin{array}{l}\text { CT: participants } \\
\text { were consecutively } \\
\text { assigned to either } \\
\text { the treatment } \\
\text { group or the } \\
\text { control group }\end{array}$ & $\begin{array}{l}\text { CT: participants } \\
\text { were either offered } \\
\text { to take part in the } \\
\text { treatment program } \\
\text { or asked to } \\
\text { participate in the } \\
\text { control group } \\
\text { during information } \\
\text { session for WLT }\end{array}$ & $\begin{array}{c}\text { RCT: randomization } \\
\text { was based on } \\
\text { generated random } \\
\text { numbers }\end{array}$ \\
\hline Intervention & $\begin{array}{l}\text { Self- help based on } \\
\text { CBT using the Swedish } \\
\text { translation of the } \\
\text { book "Overcoming } \\
\text { Binge Eating" }\end{array}$ & $\begin{array}{l}\text { Email therapy based } \\
\text { on CBT working on } \\
\text { a model of the } \\
\text { eating disorder }\end{array}$ & $\begin{array}{l}20 \text { minutes writings } \\
\text { on the basis of the } \\
\text { Pennebaker task }\end{array}$ & $\begin{array}{l}\text { Guided self-help } \\
\text { treatment program } \\
\text { consisting of } 11 \\
\text { modules based on } \\
\text { CBT targeting } \\
\text { behavioural and } \\
\text { psychological } \\
\text { aspects of BED }\end{array}$ & $\begin{array}{l}\text { guided self-help } \\
\text { treatment program } \\
\text { "Overcoming } \\
\text { Bulimia Online" } \\
\text { consisting of eight } \\
\text { sessions based on } \\
\text { CBT }\end{array}$ & $\begin{array}{l}\text { Guided self-help } \\
\text { program } \\
\text { introducing CBT } \\
\text { and } \\
\text { psychoeducational } \\
\text { concepts in seven } \\
\text { sequential stepsc }\end{array}$ & $\begin{array}{l}\text { Guided self-help } \\
\text { program and } \\
\text { consisting of } 11 \\
\text { modules based on } \\
\text { CBT targeting } \\
\text { behavioural and } \\
\text { psychological } \\
\text { aspects of BED }\end{array}$ & $\begin{array}{l}\text { Guided self-help } \\
\text { program referring to } \\
\text { CBT main principles } \\
\text { for treatment of BN }\end{array}$ \\
\hline Anonymity & $\begin{array}{l}\text { No: EDE interview was } \\
\text { performed as main } \\
\text { assessment }\end{array}$ & $\begin{array}{l}\text { Yes: no interview or } \\
\text { face-to-face } \\
\text { meeting was } \\
\text { conducted }\end{array}$ & $\begin{array}{l}\text { Yes: no interview or } \\
\text { face-to-face } \\
\text { meeting was } \\
\text { conducted }\end{array}$ & $\begin{array}{c}\text { three additional } \\
\text { face-to-face } \\
\text { evaluations during a } \\
\text { year }\end{array}$ & $\begin{array}{l}\text { introduction in a } \\
\text { face-to-face } \\
\text { meeting or as } \\
\text { telephone } \\
\text { assessment }\end{array}$ & $\begin{array}{l}\text { No: two face-to-face } \\
\text { evaluations with } \\
\text { their coaches } \\
\text { during therapy }\end{array}$ & $\begin{array}{l}\text { no: three additional } \\
\text { face-to-face } \\
\text { evaluations }\end{array}$ & $\begin{array}{l}\text { Yes: only an interview } \\
\text { with a diagnostician }\end{array}$ \\
\hline Lengths & 12 weeks & Three months & Three days & Six months & 8 to 12 weeks & Four months & Six months & 20 weeks \\
\hline $\begin{array}{l}\text { Frequency of } \\
\text { contact }\end{array}$ & $\begin{array}{l}\text { One to two email } \\
\text { contacts per week }\end{array}$ & $\begin{array}{l}\text { On average two } \\
\text { emails per week } \\
\text { were expected }\end{array}$ & $\begin{array}{l}\text { No contact at all } \\
\text { during therapy }\end{array}$ & $\begin{array}{l}\text { Weekly e-mail } \\
\text { contact during the } \\
\text { intervention phase; } \\
\text { monthly e-mail } \\
\text { contact during the } \\
\text { follow-up period }\end{array}$ & $\begin{array}{l}\text { Therapists sent } \\
\text { emails once every } \\
\text { one to two weeks } \\
\text { and responded to } \\
\text { any email received }\end{array}$ & $\begin{array}{l}\text { Weekly e-mail } \\
\text { contact with their } \\
\text { coach during } \\
\text { intervention phase }\end{array}$ & $\begin{array}{l}\text { Therapists sent } \\
\text { weekly e-mails and } \\
\text { participants had to } \\
\text { write at least one } \\
\text { email each week to } \\
\text { their coaches }\end{array}$ & $\begin{array}{l}\text { Treatment includes } 25 \\
\text { scheduled therapist } \\
\text { feedback moments }\end{array}$ \\
\hline $\begin{array}{l}\text { Number and } \\
\text { diagnosis of } \\
\text { participants }\end{array}$ & $\begin{array}{l}73 \text { participants with } \\
\text { full or sub-threshold } \\
\text { Bulimia Nervosa (BN) } \\
\text { or Binge Eating } \\
\text { Disorder (BED) } \\
\text { diagnosis according to } \\
\text { DSM-IV; sub-threshold } \\
\text { BN was defined as } \\
\text { episodes of binge } \\
\text { eating and } \\
\text { compensatory } \\
\text { behaviour at least } \\
\text { twice-monthly for the } \\
\text { last three months. BED } \\
\text { participants needed to } \\
\text { have at least two OBEs } \\
\text { per month for the last } \\
\text { six months }\end{array}$ & $\begin{array}{c}97 \text { participants } \\
\text { suffering from BN } \\
\text { ( } \mathrm{n}=36 \text { purging; } \\
\mathrm{n}=15 \text { non-purging), } \\
\text { BED }(\mathrm{n}=26) \text { or } \\
\text { EDNOS }(\mathrm{n}=20) \\
\text { diagnosis according } \\
\text { to DSM-IV }\end{array}$ & $\begin{array}{l}94 \text { participants } \\
\text { suffering from BN; } \\
\text { Participants were } \\
\text { required to score at } \\
\text { or above the } \\
\text { medium-range cut } \\
\text { off for bulimic } \\
\text { symptomatology }\end{array}$ & $\begin{array}{l}74 \text { participants } \\
\text { suffering from BED } \\
\text { ( } \mathrm{n}=43 \text { ) or sub- } \\
\text { threshold BED } \\
(\mathrm{n}=31 \text { ) diagnosis } \\
\text { according to DSM- } \\
\text { IV; for sub-threshold } \\
\text { BED participants } \\
\text { needed to have at } \\
\text { least one OBE } \\
\text { weekly for the last } \\
\text { three months }\end{array}$ & $\begin{array}{c}76 \text { students } \\
\text { suffering from BN } \\
(n=39) \text { or EDNOS } \\
(n=37) \text { diagnosis } \\
\text { according to DSM- } \\
\text { IV; persons suffering } \\
\text { from BED were } \\
\text { excluded }\end{array}$ & $\begin{array}{c}62 \text { participants } \\
\text { suffering from BN } \\
\text { purging subtype; } \\
\text { diagnosis according } \\
\text { to DSM-IV }\end{array}$ & $\begin{array}{c}42 \text { obese } \\
\text { participants } \\
\text { suffering from BED } \\
(\mathrm{n}=21) \text { or sub- } \\
\text { threshold BED } \\
(\mathrm{n}=21) \text {; diagnosis } \\
\text { according to DSM- } \\
\mathrm{IV} ; \text { Frequency of } \\
\text { binges had to be at } \\
\text { least for once a } \\
\text { month during the } \\
\text { last three months }\end{array}$ & $\begin{array}{c}105 \text { participants } \\
\text { suffering from BN } \\
\text { symptoms ( } 80 \% \\
\text { engaged in purging } \\
\text { behaviour) } \\
\text { A formal diagnosis of } \\
\text { BN was not an } \\
\text { inclusion criteria. } \\
\text { Participants had to } \\
\text { report recurrent binge } \\
\text { eating, inappropriate } \\
\text { weight-control } \\
\text { behaviour and } \\
\text { elevated concern with } \\
\text { body shape and } \\
\text { weight }\end{array}$ \\
\hline
\end{tabular}


Table 2 Characteristics of treatment programs applied in included studies (Continued)

\begin{tabular}{|c|c|c|c|c|c|c|c|c|}
\hline Women N (\%) & 69 (97.3\%) & 93 (95.9\%) & $71(75.5 \%)$ & $74(100 \%)$ & 75 (98.7\%) & $62(100 \%)$ & $42(100 \%)$ & 104 (99\%) \\
\hline \multirow{3}{*}{$\begin{array}{l}\text { Age of } \\
\text { participants } \\
M(\text { SD) }\end{array}$} & $\begin{array}{c}\text { Intervention Group: } \\
35.5(11.4)\end{array}$ & $\begin{array}{l}\text { Whole sample: } \\
24.5(23-25.9)\end{array}$ & $\begin{array}{c}\text { Whole sample: } \\
28.9 \text { (9.8) }\end{array}$ & $\begin{array}{l}\text { Whole sample: } \\
36(11.4)\end{array}$ & $\begin{array}{c}\text { Whole sample: } \\
23.9 \text { (5.69) }\end{array}$ & $\begin{array}{c}\text { Whole sample: } \\
23.7 \text { (3.6) }\end{array}$ & $\begin{array}{c}\text { Intervention Group: } \\
44.6(11.4)\end{array}$ & $\begin{array}{l}\text { Online-CBT: } \\
30(10)\end{array}$ \\
\hline & $\begin{array}{l}\text { Control group: } \\
33.7(9.3)\end{array}$ & & & & & & $\begin{array}{l}\text { Control group: } \\
41.0(8.2)\end{array}$ & $\begin{array}{l}\text { Bibliotherapy: } \\
31(9)\end{array}$ \\
\hline & & & & & & & & $\begin{array}{l}\text { Waiting list group: } \\
\quad 32(11)\end{array}$ \\
\hline
\end{tabular}

RCT Randomized Controlled Trial, CT Controlled Trial, CBT Cognitive Behavioural Therapy, EDE Eating Disorder Examination, BN Bulimia Nervosa, BED Binge Eating Disorder, EDNOS Eating Disorder Not Other Specified. 
Table 3 Control group characteristics, Outcome measures, time points of assessment, dropout and abstinence rates of included studies

\begin{tabular}{|c|c|c|c|c|c|c|c|c|}
\hline Authors & Ljotsson et al. [16] & $\begin{array}{l}\text { Robinson \& } \\
\text { Serfaty [17] }\end{array}$ & $\begin{array}{c}\text { Johnston et al. } \\
{[15]}\end{array}$ & Carrard et al. [12] & $\begin{array}{l}\text { Sanchez-Ortiz et } \\
\text { al. [19] }\end{array}$ & $\begin{array}{c}\text { Fernandez-Aranda } \\
\text { et al. [14] }\end{array}$ & Carrard et al. [13] & Ruwaard et al. [18] \\
\hline Control group & $\begin{array}{l}\text { Waiting list }(n=36) \\
\text { with assessment at } \\
\text { mid-treatment and } \\
\text { short weekly reports } \\
\text { on eating behaviour }\end{array}$ & $\begin{array}{l}\text { Unsupported self } \\
\text { directed writing } \\
(n=34) \text {. Waiting list } \\
\text { control }(n=27)\end{array}$ & $\begin{array}{l}\text { Superficial writing } \\
\text { control group } \\
(n=46)\end{array}$ & $\begin{array}{l}\text { Waiting list control } \\
\text { group }(n=37) \text { with } \\
\text { monthly email } \\
\text { contact during } \\
\text { waiting period }\end{array}$ & $\begin{array}{l}\text { Waiting list control } \\
\text { group }(n=38)\end{array}$ & $\begin{array}{l}\text { Waiting list }(\mathrm{n}=31) \\
\text { for } 12 \text { weeks } \\
\text { keeping a food } \\
\text { diary, recording } \\
\text { binge eating and } \\
\text { purging episodes }\end{array}$ & $\begin{array}{l}\text { Waiting list control } \\
\text { group }(n=20), \\
\text { waiting for a weight } \\
\text { loss treatment }\end{array}$ & $\begin{array}{l}\text { Waiting list control } \\
\text { group }(\mathrm{n}=35) \text {. } \\
\text { Bibliotherapy } \\
(\mathrm{n}=35)\end{array}$ \\
\hline \multirow{3}{*}{ Measures } & EDE-Q & BITE & BITE & EDE-Q & EDE & EDI & EDE-Q & EDE-Q \\
\hline & EDI-2 & & & EDI-2 & & BITE & & \\
\hline & & & & & & Food Diary & & \\
\hline \multirow{3}{*}{ Assessments } & Pre-treatment & Baseline & Baseline & Baseline & Baseline & Baseline & Baseline & Baseline \\
\hline & Post-treatment & Post-treatment & $\begin{array}{c}\text { Four weeks follow- } \\
\text { up }\end{array}$ & Post-treatment & Post-treatment & Post-treatment & Post-tretament & Post-tretament \\
\hline & Six month follow-up & & $\begin{array}{c}\text { Eight weeks follow- } \\
\text { up }\end{array}$ & Six month follow-up & $\begin{array}{l}\text { Three month } \\
\text { follow-up }\end{array}$ & & Six month follow-up & 12 month follow-up \\
\hline Treatment dropout & $31.4 \%$ & $47.2 \%$ & $16.7 \%$ & $24.3 \%$ & $21.1 \%$ & $45.0 \%$ & $9 \%$ & $25.7 \%$ \\
\hline Study dropout & $2.9 \%$ & $37.1 \%$ & $14.9 \%$ & $17.6 \%$ & $11.8 \%$ & n.a. & $4.8 \%$ & $21.0 \%$ \\
\hline $\begin{array}{l}\text { Definition of } \\
\text { abstinence }\end{array}$ & $\begin{array}{l}\text { no episodes of } \\
\text { binge eating or } \\
\text { purging during the } \\
28 \text { days prior to the } \\
\text { post-treatment } \\
\text { assessment }\end{array}$ & $\begin{array}{l}\text { Criteria for an eating } \\
\text { disorder are no } \\
\text { longer fulfilled }\end{array}$ & $\begin{array}{l}\text { Participants not } \\
\text { falling within the } \\
\text { clinical range of the } \\
\text { BITE total score }\end{array}$ & $\begin{array}{l}\text { Abstinence from } \\
\text { OBEs as measured } \\
\text { by the EDE-Q for } \\
\text { the last } 28 \text { days }\end{array}$ & $\begin{array}{l}\text { Lack of vomiting } \\
\text { and laxative abuse } \\
\text { abstinence from } \\
\text { OBEs over a month } \\
\text { long period }\end{array}$ & $\begin{array}{c}\text { Abstinence of } \\
\text { binges and vomits: } \\
\text { no information } \\
\text { about duration } \\
\text { available }\end{array}$ & $\begin{array}{c}\text { No binge episode } \\
\text { over the last three } \\
\text { months }\end{array}$ & $\begin{array}{c}\text { No binge or } \\
\text { purging episode } \\
\text { over the last } 28 \\
\text { days according to } \\
\text { EDE-Q }\end{array}$ \\
\hline \multirow{2}{*}{$\begin{array}{l}\text { Rate of abstinence } \\
\text { (Intervention } \\
\text { group) }\end{array}$} & $37 \%$ & $23.5 \%$ & $\begin{array}{c}23.7 \% \text { (at baseline) } \\
\text { to } 35 \% \text { (at } 8 \text { week } \\
\text { follow-up) }\end{array}$ & $35.1 \%$ & $25.8 \%$ & $22.6 \%$ & $45 \%$ & $\begin{array}{c}\text { Bingeing: } 57 \% \text { (at } \\
\text { baseline) to } 94 \% \text { at } \\
\text { post-treatment }\end{array}$ \\
\hline & & & & & & & & $\begin{array}{c}\text { Purging: } 60 \% \text { (at } \\
\text { baseline) to } 100 \% \text { at } \\
\text { post-treatment }\end{array}$ \\
\hline \multirow{2}{*}{$\begin{array}{l}\text { Rate of abstinence } \\
\text { (Control group) }\end{array}$} & $15 \%$ & $0.0 \%$ & n.a. & $8.1 \%$ & $13.9 \%$ & $0 \%$ & $15 \%$ & $\begin{array}{c}\text { Bingeing: } 77 \% \\
\text { (at baseline) to } 86 \% \\
\text { at post-treatment }\end{array}$ \\
\hline & & & & & & & & $\begin{array}{l}\text { Purging: } 83 \% \\
\text { (at baseline) to } 89 \% \\
\text { at post-treatment }\end{array}$ \\
\hline
\end{tabular}

EDE-Q Eating Disorder Examination Questionnaire, EDE Eating Disorder Examination, EDI-2 Eating Disorder Inventory - 2, BITE Bulimia Investigatory Test Edinburgh.

OBE Objectivte Binge Episodes as assessed with the Eating Disorder Examination Questionnaire or the Eating Disorder Examination Interview. 
Table 4 Effect sizes and confidence intervals of primary and secondary outcomes

\begin{tabular}{|c|c|c|c|c|c|c|c|c|}
\hline & & Ljotsson et al. [16] & Johnston et al. [15] & Carrard et al. [12] & Sanchez-Ortiz et al. [19] & $\begin{array}{c}\text { Fernandez-Aranda } \\
\text { et al. [14] }\end{array}$ & Carrard et al. [13] & Ruwaard et al. [18] \\
\hline \multicolumn{9}{|l|}{ Primary outcome } \\
\hline \multirow{2}{*}{ Binge episodes } & $\mathrm{ES}_{\text {within }}(\mathrm{Cl}$ 95\%) & $0.98(0.58-1.38)$ & & $1.05(0.65-1.45)$ & $0.75(0.39-1.11)$ & & $0.90(0.40-1.40)$ & $1.05(0.63-1.47)$ \\
\hline & $\mathrm{ES}_{\text {between }}(\mathrm{Cl}$ 95\%) & $0.52(0.04-1.00)$ & & $0.52(0.06-0.98)$ & $0.35(-0.10-0.80)$ & 0.49 & $0.20(-0.41-0.81)$ & $0.43(-0.04-0.90)$ \\
\hline \multirow{2}{*}{ Purging } & $\mathrm{ES}_{\text {within }}(\mathrm{Cl}$ 95\%) & $0.41(0.06-0.76)$ & & & $0.56(0.22-0.90)$ & & & $0.77(0.39-1.15)$ \\
\hline & $\mathrm{ES}_{\text {between }}(\mathrm{Cl} 95 \%)$ & $1.63(1.08-2.18)$ & & & $0.27(-0.18-0.72)$ & & & $0.45(-0.02-0.92)$ \\
\hline \multirow{2}{*}{ Vomiting } & $\mathrm{ES}_{\text {within }}(\mathrm{Cl}$ 95\%) & & & & $0.46(0.12-0.80)$ & & & \\
\hline & $\mathrm{ES}_{\text {between }}(\mathrm{Cl} 95 \%)$ & & & & $0.29(-0.16-0.74)$ & 0.78 & & \\
\hline \multicolumn{9}{|l|}{ Secondary Outcome } \\
\hline \multicolumn{9}{|l|}{ EDE-Q } \\
\hline \multirow{2}{*}{ Restraint } & $E S_{\text {within }}(\mathrm{Cl} 95 \%)$ & $0.32(-0.02-0.66)$ & & $0.47(0.13-0.81)$ & $1.26(0.83-1.69)$ & & $0.23(-0.19-0.65)$ & \\
\hline & $\mathrm{ES}_{\text {between }}(\mathrm{Cl}$ 95\%) & $0.26(-0.21-0.73)$ & & $0.14(-0.32-0.60)$ & $0.79(0.32-1.26)$ & & $0.24(-0.37-0.85)$ & \\
\hline \multirow{2}{*}{ Eating concern } & $\mathrm{ES}_{\text {within }}(\mathrm{Cl}$ 95\%) & $0.86(0.47-1.25)$ & & & $1.05(0.65-1.45)$ & & $0.78(0.30-1.26)$ & \\
\hline & $\mathrm{ES}_{\text {between }}(\mathrm{Cl} 95 \%)$ & $0.99(0.49-1.49)$ & & & $0.84(0.37-1.31)$ & & $0.39(-0.22-1.00)$ & \\
\hline \multirow{2}{*}{ Shape concern } & $\mathrm{ES}_{\text {within }}(\mathrm{Cl} 95 \%)$ & $0.51(0.16-0.86)$ & & $0.89(0.51-1.27)$ & $1.02(0.63-1.41)$ & & $1.02(0.50-1.54)$ & \\
\hline & $\mathrm{ES}_{\text {between }}(\mathrm{Cl} 95 \%)$ & $0.92(0.42-1.42)$ & & $0.28(-0.18-0.74)$ & $1.05(0.57-1.53)$ & & $0.58(-0.04-1.20)$ & \\
\hline \multirow{2}{*}{ Weight concern } & $\mathrm{ES}_{\text {within }}(\mathrm{Cl}$ 95\%) & $0.78(0.40-1.16)$ & & & $0.73(0.37-1.09)$ & & $1.11(0.57-1.65)$ & \\
\hline & $\mathrm{ES}_{\text {between }}(\mathrm{Cl}$ 95\%) & $1.10(0.59-1.61)$ & & & $0.79(0.32-1.26)$ & & $0.18(-0.43-0.79)$ & \\
\hline \multirow{2}{*}{ Total } & $\mathrm{ES}_{\text {within }}(\mathrm{Cl}$ 95\%) & $0.71(0.34-1.08)$ & & $1.18(0.76-1.60)$ & $1.21(0.79-1.63)$ & & $0.79(0.31-1.27)$ & $1.18(0.75-1.61)$ \\
\hline & $\mathrm{ES}_{\text {between }}(\mathrm{Cl}$ 95\%) & $0.96(0.46-1.46)$ & & $0.45(-0.01-0.91)$ & $1.09(0.61-1.57)$ & & $0.32(-0.29-0.93)$ & $0.50(0.02-0.98)$ \\
\hline \multicolumn{9}{|l|}{ EDI (-2) } \\
\hline \multirow{2}{*}{ Drive for thinness } & $\mathrm{ES}_{\text {within }}(\mathrm{Cl}$ 95\%) & $0.55(0.19-0.91)$ & & $0.62(0.27-0.97)$ & & & & \\
\hline & $\mathrm{ES}_{\text {between }}(\mathrm{Cl} 95 \%)$ & $0.99(0.49-1.49)$ & & $0.48(0.02-0.94)$ & & 0.25 & & \\
\hline \multirow{2}{*}{ Body dissatisfaction } & $\mathrm{ES}_{\text {within }}(\mathrm{Cl}$ 95\%) & $0.39(0.05-0.73)$ & & $0.60(0.25-0.95)$ & & & & \\
\hline & $\mathrm{ES}_{\text {between }}(\mathrm{Cl}$ 95\%) & $0.76(0.27-1.25)$ & & $0.05(-0.41-0.51)$ & & 0.16 & & \\
\hline \multirow{2}{*}{ Interoceptive awareness } & $\mathrm{ES}_{\text {within }}(\mathrm{Cl}$ 95\%) & $0.70(0.33-1.07)$ & & $0.53(0.18-0.88)$ & & & & \\
\hline & $\mathrm{ES}_{\text {between }}(\mathrm{Cl}$ 95\%) & $0.88(0.39-1.37)$ & & $0.46(0.00-0.92)$ & & 0.03 & & \\
\hline \multirow{2}{*}{ Bulimia } & $\mathrm{ES}_{\text {within }}(\mathrm{Cl}$ 95\%) & $0.79(0.41-1.17)$ & & $1.56(1.08-2.04)$ & & & & \\
\hline & $\mathrm{ES}_{\text {between }}(\mathrm{Cl}$ 95\%) & $0.94(0.44-1.44)$ & & $1.08(0.59-1.57)$ & & 0.54 & & \\
\hline \multirow{2}{*}{ Interpersonal distrust } & $\mathrm{ES}_{\text {within }}(\mathrm{Cl}$ 95\%) & $0.02(-0.31-0.35)$ & & $0.21(-0.12-0.54)$ & & & & \\
\hline & $\mathrm{ES}_{\text {between }}(\mathrm{Cl}$ 95\%) & $0.00(-0.47-0.47)$ & & $0.17(-0.29-0.63)$ & & 0.32 & & \\
\hline \multirow{2}{*}{ Ineffectiveness } & $\mathrm{ES}_{\text {within }}(\mathrm{Cl}$ 95\%) & $0.47(0.12-0.82)$ & & $0.36(0.03-0.69)$ & & & & \\
\hline & $\mathrm{ES}_{\text {between }}(\mathrm{Cl}$ 95\%) & $0.57(0.09-1.05)$ & & $0.33(-0.13-0.79)$ & & 0.05 & & \\
\hline
\end{tabular}


Table 4 Effect sizes and confidence intervals of primary and secondary outcomes (Continued)

\begin{tabular}{|c|c|c|c|c|}
\hline \multirow{2}{*}{ Maturity fears } & \multirow{2}{*}{\multicolumn{2}{|c|}{$\begin{array}{l}\mathrm{ES}_{\text {within }}(\mathrm{Cl} \text { 95\%) } \\
\mathrm{ES}_{\text {between }}(\mathrm{Cl} 95 \%)\end{array}$}} & $0.23(-0.10-0.56)$ & \multirow[b]{2}{*}{0.63} \\
\hline & & & $0.15(-0.31-0.61)$ & \\
\hline \multirow{2}{*}{ Perfectionism } & $\mathrm{ES}_{\text {within }}(\mathrm{Cl}$ 95\%) & $0.21(-0.13-0.55)$ & $0.12(-0.20-0.44)$ & \\
\hline & $\mathrm{ES}_{\text {between }}(\mathrm{Cl}$ 95\%) & $0.22(-0.25-0.69)$ & $0.19(-0.27-0.65)$ & 0.13 \\
\hline \multirow{2}{*}{ Impulse regulation } & $\mathrm{ES}_{\text {within }}(\mathrm{Cl}$ 95\%) & & $0.51(0.17-0.85)$ & \\
\hline & $\mathrm{ES}_{\text {between }}(\mathrm{Cl} 95 \%)$ & & $0.31(-0.15-0.77)$ & \\
\hline \multirow{2}{*}{ Social insecurity } & $\mathrm{ES}_{\text {within }}(\mathrm{Cl}$ 95\%) & & $0.64(0.29-0.99)$ & \\
\hline & $\mathrm{ES}_{\text {between }}(\mathrm{Cl}$ 95\%) & & $0.27(-0.19-0.73)$ & \\
\hline Total & $\mathrm{ES}_{\text {between }}(\mathrm{Cl} 95 \%)$ & & & 0.37 \\
\hline \multicolumn{5}{|l|}{ BITE } \\
\hline \multirow{2}{*}{ Severity } & $\mathrm{ES}_{\text {within }}(\mathrm{Cl}$ 95\%) & & $0.14(-0.14-0.42)$ & \\
\hline & $\mathrm{ES}_{\text {between }}(\mathrm{Cl}$ 95\%) & & $0.28(-0.13-0.69)$ & \\
\hline \multirow{2}{*}{ Symptom } & $\mathrm{ES}_{\text {within }}(\mathrm{Cl}$ 95\%) & & $0.10(-0.18-0.38)$ & \\
\hline & $\mathrm{ES}_{\text {between }}(\mathrm{Cl}$ 95\%) & & $0.03(-0.37-0.43)$ & \\
\hline \multirow{2}{*}{ Total } & $\mathrm{ES}_{\text {within }}(\mathrm{Cl}$ 95\%) & & $0.14(-0.14-0.42)$ & \\
\hline & $\mathrm{ES}_{\text {between }}(\mathrm{Cl}$ 95\%) & & $0.13(-0.28-0.54)$ & 1.03 \\
\hline
\end{tabular}

ES Effect Sizes calculated according to Hedges [40], Cl Confidence intervals were calculated using formulas according to Hedges and Olkin [40]. Boldface data show Cls not covering zero. EDE-Q Eating Disorder Questionnaire, EDI-2 Eating Disorder Inventory - 2, BITE Bulimia Investigatory Test Edinburgh. 


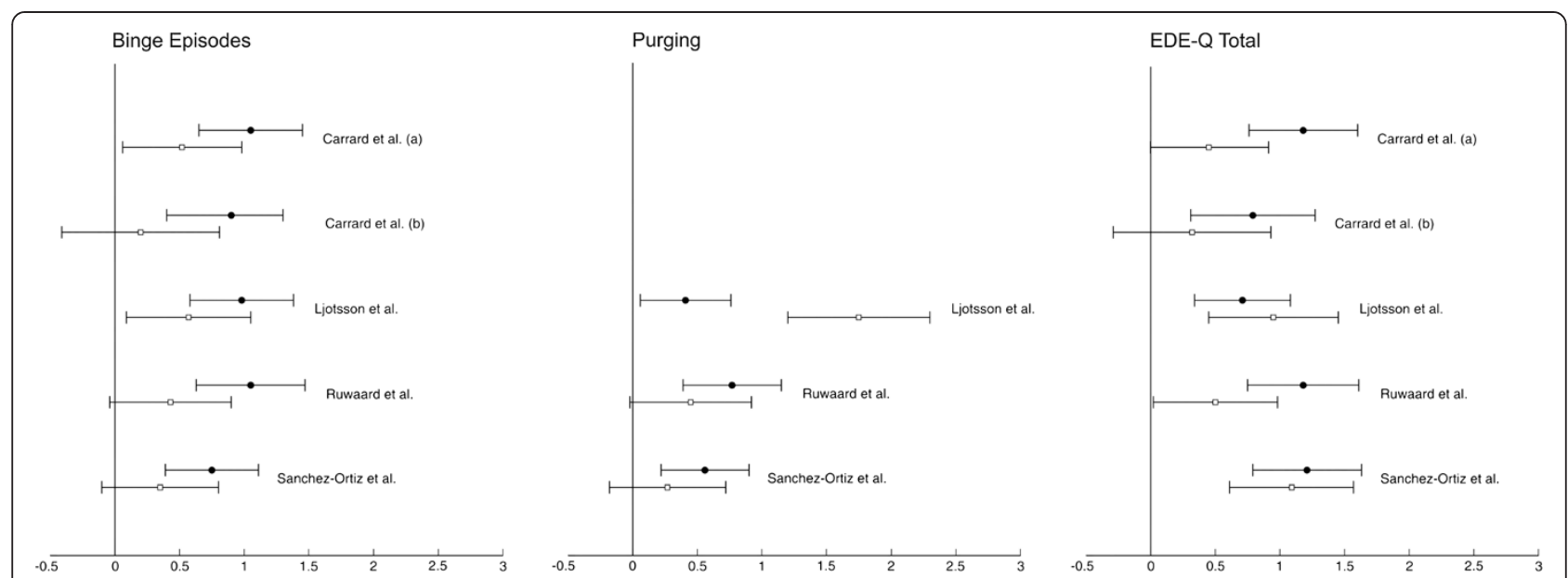

Figure 2 Effect sizes and corresponding confidence intervals for Bingeing, Purging and the EDE-Q Total Score.

defined time period, in two studies abstinence was defined as no longer falling within the clinical range of the BITE [15] or no longer fulfilling criteria for an eating disorder according to the DSM-IV [17]. All of the studies found a higher rate of abstinence in the intervention group compared to the control group with lowest contact, but this difference was only tested for significance in four studies $[12,13,17,18]$. All of these analyses reached statistical significance.

\section{Secondary outcomes}

Figure 2 also shows effect sizes and corresponding confidence intervals for EDE-Q total. Regarding secondary outcomes, as assessed with the questionnaires described above, the included studies found high effects from preto post-treatment in the EDE-Q total, as assessed in five studies $[12,13,16,18,19]$. Positive results of the intervention were also found for the subscales of the EDE-Q, where assessed. When groups were compared for EDE$\mathrm{Q}$ total, moderate to high effect sizes were found in three studies $[16,18,19]$, while in the two studies of Carrard and colleagues [12,13] these effect sizes were only small to moderate, as were the results of the EDE$Q$ subscales. In the remaining two studies assessing EDEQ subscales [16,19], effect sizes between groups were high with the one exception in the "Restraint" subscale, that was only significant in the study conducted by SanchezOrtiz et al. [19]. In two studies [12,16] the EDI-2 was implemented in addition to the EDE-Q to aid treatment outcome assessment. In both studies medium to high effects of the intervention were found from pre- to posttreatment for most of the subscales, reinforcing the results reported for the EDE-Q. These studies also showed medium to high effect sizes on some subscales of the EDI2 when groups were compared. Additionally, in the study by Fernandez-Aranda [14], the effect sizes reported were small to medium. In two of the studies - those conducted by Robinson and Serfaty [17] and Johnston et al. [15] eating disorder symptoms were not assessed with either the EDE-Q or the EDI but with the Bulimia Investigatory Test Edinburgh (BITE) [39]. No significant treatment effects either within or between the treatment groups could be shown in the study of Johnston et al. [15]. In the study of Robinson and Serfaty [17], only changes within the whole group of participants were examined. For this sample, no significant changes in questionnaire scores were found.

\section{Dropout rates}

As authors defined dropout differently between studies, treatment dropouts were considered separately from study dropouts and rates are displayed in Table 3. Treatment dropouts were intervention group participants who did not complete the treatment, whereas study dropouts were participants from either group who did not fill out the post-treatment assessment. While treatment dropout rates were between 9\% [13] and 47.2\% [17], study dropouts ranged between $2.9 \%$ [16] and $37.1 \%$ [17]. In this study, no information was given about whether the number of participants, who did not complete treatment, differed from the number of participants, who did not fill out post-assessment, meaning that the treatment dropout we report here is identical to study dropout in the treatment group. In two studies $[12,19]$ treatment dropouts referred to participants who did not use treatment at all or did not finish the first module of treatment.

\section{Stability of results}

Of the six studies that included follow-up measures $[12,13,15,16,18,19]$, the one with the 8-week followup failed to find stable treatment effects [15]. The remaining five studies found that the results achieved at post-treatment were stable or even improved over the follow-up period. 


\section{Additional outcomes}

Table 5 gives an overview of the additional results. In two studies [14,18], neither depression nor any other additional outcome was explicitly assessed. All of the remaining studies assessed depression: three $[12,13,17]$ utilising the Beck Depression Inventory (BDI) [42], or
BDI-II [43], two studies [15,19] making use of the Hospital Anxiety and Depression Scale (HADS) $[44,45]$ and one study [16] using the Montgomery Åsberg Depression Scale Self-assessment (MADRS) [46]. Divergent results were found between the studies. Again, in the study by Robinson and Serfaty [17], no information for

Table 5 Effect sizes and confidence intervals of questionnaires regarding additional outcomes

\begin{tabular}{|c|c|c|c|c|c|c|}
\hline & & Ljotsson et al. [16] & Johnston et al. [15] & Carrard et al. [12] & $\begin{array}{l}\text { Sanchez-Ortiz } \\
\text { et al. [19] }\end{array}$ & Carrard et al. [13] \\
\hline \multicolumn{7}{|l|}{ Depression } \\
\hline \multirow{2}{*}{ BDI (-II) } & $\mathrm{ES}_{\text {within }}(\mathrm{Cl} 95 \%)$ & & & $0.61(0.26-0.96)$ & & $0.61(0.15-1.07)$ \\
\hline & $\mathrm{ES}_{\text {between }}(\mathrm{Cl}$ 95\%) & & & $0.37(-0.09-0.83)$ & & $0.80(0.17-1.43)$ \\
\hline \multirow{2}{*}{ HADS } & $\mathrm{ES}_{\text {within }}(\mathrm{Cl} 95 \%)$ & & $0.08(-0.20-0.36)$ & & $2.18(1.59-2.77)$ & \\
\hline & $\mathrm{ES}_{\text {between }}(\mathrm{Cl}$ 95\%) & & $0.18(-0.23-0.59)$ & & $0.97(0.49-1.45)$ & \\
\hline \multirow{2}{*}{ MADRS } & $\mathrm{ES}_{\text {within }}(\mathrm{Cl} 95 \%)$ & $0.78(0.40-1.16)$ & & & & \\
\hline & $\mathrm{ES}_{\text {between }}(\mathrm{Cl}$ 95\%) & $0.76(0.27-1.14)$ & & & & \\
\hline \multicolumn{7}{|l|}{ Anxiety } \\
\hline \multirow{2}{*}{ HADS } & $\mathrm{ES}_{\text {within }}(\mathrm{Cl}$ 95\%) & & $0.27(-0.02-0.56)$ & & $1.01(0.62-1.40)$ & \\
\hline & $\mathrm{ES}_{\text {between }}(\mathrm{Cl}$ 95\%) & & $0.02(-0.38-0.42)$ & & $0.82(0.33-1.31)$ & \\
\hline \multicolumn{7}{|l|}{ Quality of life } \\
\hline \multicolumn{7}{|l|}{ IWQOL-Lite } \\
\hline \multirow{2}{*}{ Physical functioning } & $\mathrm{ES}_{\text {within }}(\mathrm{Cl} 95 \%)$ & & & & & $0.44(0.00-0.88)$ \\
\hline & $\mathrm{ES}_{\text {between }}(\mathrm{Cl}$ 95\%) & & & & & $0.37(-0.24-0.98)$ \\
\hline \multirow{2}{*}{ Self-esteem } & $\mathrm{ES}_{\text {within }}(\mathrm{Cl} 95 \%)$ & & & & & $0.55(0.10-1.00)$ \\
\hline & $\mathrm{ES}_{\text {between }}(\mathrm{Cl}$ 95\%) & & & & & $0.59(-0.03-1.21)$ \\
\hline \multirow{2}{*}{ Sexual life } & $\mathrm{ES}_{\text {within }}(\mathrm{Cl} 95 \%)$ & & & & & $0.49(0.05-0.93)$ \\
\hline & $\mathrm{ES}_{\text {between }}(\mathrm{Cl}$ 95\%) & & & & & $0.84(0.21-1.47)$ \\
\hline \multirow{2}{*}{ Public distress } & $\mathrm{ES}_{\text {within }}(\mathrm{Cl} 95 \%)$ & & & & & $0.52(0.07-0.97)$ \\
\hline & $\mathrm{ES}_{\text {between }}(\mathrm{Cl} 95 \%)$ & & & & & $0.73(0.10-1.36)$ \\
\hline \multirow{2}{*}{ Work } & $\mathrm{ES}_{\text {within }}(\mathrm{Cl} 95 \%)$ & & & & & $0.43(-0.01-0.87)$ \\
\hline & $\mathrm{ES}_{\text {between }}(\mathrm{Cl}$ 95\%) & & & & & $0.94(0.30-1.58)$ \\
\hline \multirow{2}{*}{ Total score } & $\mathrm{ES}_{\text {within }}(\mathrm{Cl} 95 \%)$ & & & $0.30(-0.03-0.63)$ & & $0.68(0.21-1.15)$ \\
\hline & $\mathrm{ES}_{\text {between }}(\mathrm{Cl}$ 95\%) & & & $0.01(-0.45-0.47)$ & & $0.78(0.15-1.41)$ \\
\hline \multicolumn{7}{|l|}{ WHOQOL-Bref } \\
\hline \multirow{2}{*}{ Physical health } & $\mathrm{ES}_{\text {within }}(\mathrm{Cl} 95 \%)$ & & & & $1.01(0.62-1.40)$ & \\
\hline & $\mathrm{ES}_{\text {between }}(\mathrm{Cl}$ 95\%) & & & & $1.03(0.55-1.51)$ & \\
\hline \multirow{2}{*}{ Psychological health } & $\mathrm{ES}_{\text {within }}(\mathrm{Cl} 95 \%)$ & & & & $0.77(0.41-1.13)$ & \\
\hline & $\mathrm{ES}_{\text {between }}(\mathrm{Cl}$ 95\%) & & & & $0.80(0.33-1.27)$ & \\
\hline \multirow{2}{*}{ Social } & $\mathrm{ES}_{\text {within }}(\mathrm{Cl} 95 \%)$ & & & & $0.67(0.32-1.02)$ & \\
\hline & $\mathrm{ES}_{\text {between }}(\mathrm{Cl}$ 95\%) & & & & $0.65(0.19-1.11)$ & \\
\hline \multirow{2}{*}{ Enviromental } & $\mathrm{ES}_{\text {within }}(\mathrm{Cl}$ 95\%) & & & & $0.15(-0.17-0.47)$ & \\
\hline & $\mathrm{ES}_{\text {between }}(\mathrm{Cl}$ 95\%) & & & & $0.19(-0.26-0.64)$ & \\
\hline \multicolumn{7}{|l|}{ SWLS } \\
\hline \multirow{2}{*}{ Total } & $\mathrm{ES}_{\text {within }}(\mathrm{Cl} 95 \%)$ & $0.41(0.06-0.76)$ & & & & \\
\hline & $\mathrm{ES}_{\text {between }}(\mathrm{Cl}$ 95\%) & $0.31(-0.17-0.79)$ & & & & \\
\hline
\end{tabular}

ES Effect Sizes calculated according to Hedges [40], Cl Confidence intervals were calculated using formulas according to Hedges and Olkin [40]. Boldface data show Cls not covering zero.

BDI (-II) Beck Depression Inventory, HADS Hospital Anxiety and Depression Scale, MADRS Montgomery Åsberg Depression Scale Self-assessment, SWLS Satisfaction with Life Scale; IWQOL-Lite, Impact of Weight on Quality of Life, WHOQOL-Bref WHO Quality of Life Questionnaire (short form). 
calculating effect sizes was available and no significant differences were found for the analysis of variance performed. In terms of effect sizes within the intervention group, four studies found medium to high effect sizes for depression $[12,13,16,19]$, while no effects were found for the three day writing task study by Johnston et al. [15]. For three of the studies [13,16,19], significant differences were also found between the groups. As they applied the HADS, two studies $[15,19]$ were additionally able to assess anxiety as an outcome variable. The study conducted by Johnston et al. [15] did not find effects within or between groups, but in the study by SanchezOrtiz et al. [19], reduction of anxiety resulted in high effect sizes both within and between the groups. Quality of life or satisfaction with life were assessed in four studies $[12,13,16,19]$. Here, medium- to high-sized effects were seen for the intervention group, with exception of one study [12]. These results did not translate to a medium to high between-group effect for the study by Ljotsson et al. [16], however.

\section{Discussion}

This review systematically evaluated the efficacy of internet-based treatment programs for different eating disorders in participants aged at least 16, based upon evidence from controlled studies. An article focusing on the effectiveness of cognitive-behavioural guided self help for eating disorders has recently been published [51], but to our knowledge this study is the first review examining the standardized effects of interventions delivered through the internet on participants suffering from different kinds of eating disorders. All studies included in this review were published in the last six years, since 2007, highlighting that this research field is relatively novel. For five of the eight studies included in this review, good methodological quality was noted [12,16-19].

All but one intervention [15] were based on CBT, which emphasizes the suitability of cognitive behavioural methods as a basis for the development of internet-based treatments for eating disorders. Of the seven studies based on CBT, six offered patients a guided self-help intervention $[12-14,16,18,19]$. Of these, one delivered the self-help intervention by book, with accompanying tasks and homework [16], while the other studies developed and used a structured treatment program. The remaining CBT-based study used e-mail therapy without following a structured treatment program [17]. All studies that were based on CBT principles and provided relevant information to calculate effect sizes found significant reductions of eating disordered behaviour within the intervention groups from pre- to post-treatment for primary outcomes (e.g. bingeing and purging) as well as secondary outcomes. These interventions were also found to be beneficial in comparison to being placed on a waiting list. The findings applied to the self-help programs implemented over the internet, but also for the self help book intervention with e-mail support and for e-mail-therapy. In summary, the results suggest that a variety of treatments, based on CBT and using the internet, can help to reduce symptoms related to eating disorders such as bulimia nervosa, binge eating and EDNOS.

There was high variance in the rates of abstinence between studies. Interestingly, the three studies with the highest rates of abstinence had the most conservative time criteria. Since these studies included patients suffering from binge eating disorder $[12,13,16]$ results are in line with previous meta-analysis findings from two other studies. In one study, the odds ratios for abstinence rates in a RCT addressing binge eating disorder significantly improved after psychotherapy and structured self-help [52]. A second study compared guided and unguided self-help for binge eating [53], where relatively high remission rates were found for OBEs. These findings can be interpreted with respect to the high spontaneous remission found for binge eating [54]. In this review, benefits of the guided self-help interventions based on CBT were superior to those elicited by the e-mail therapy [17] or the three day writing task [15].

Additionally, effects on secondary outcomes, as assessed by questionnaires, were comparable with those produced by face-to-face therapies. For example, a meta-analysis examining the effects of different treatments for binge eating [52] found medium to high effect sizes for psychotherapy as well as structured self-help, both mainly based on CBT. These results are in line with significant medium to high effect sizes found in the studies included in this review that addressed patients suffering from BED $[12,13,16]$. Furthermore, a meta-analysis of studies that used CBT in face-to-face treatment for bulimia nervosa found effect sizes ranging from -0.03 to 1.00 for behavioural measures and from 0.26 to 0.98 for cognitive measures [55]. Again, these results are in line with results found in the CBT-based intervention for BN of studies included in the review. Additionally, follow-up examination results indicated that the effects of the treatments did not decrease over time, implying long-term stability of these positive effects of CBT-based guided self-help interventions for eating disorders delivered over the internet. The two studies that did not find significant effects or stable treatment results either within or between groups were those by Johnston et al. [15] and Robinson and Serfaty [17]. Neither study utilized structured self-help based upon CBT principles, instead using e-mail therapy [17] or a writing task [15]. Furthermore, the study by Johnston et al. [15] differed from the other studies in terms of lengths and therapeutic contact, and one could argue that the focus was not the internet as a tool, but rather evaluation of an intervention paradigm which 
had not previously been assessed for the treatment of eating disorders.

To further evaluate internet-based interventions for the treatment of eating disorders, dropout rates should be taken into account. The relative numbers of participants who did not finish treatment (treatment dropout) differed between the studies included and were higher in studies that included participants suffering from symptoms of BN $[14,16,18,19]$. This matches results from face-to-face therapies, where high treatment dropout rates have also been documented. While Garner [56] reported a mean dropout rate of $15.3 \%$ in their review of CBT for the treatment of bulimia nervosa, the dropout rates reported in controlled studies are assumed to be an underestimate of the rates in a general clinical setting [57-61]. One possible explanation for the different dropout rates that were found in internet-based interventions of depression and anxiety [62] is the level of anonymity. But, contrary to this finding, no clear connection between anonymity and treatment dropout was seen in the present review. The studies providing anonymity were not necessarily those where high treatment dropout rates were reported. Instead of anonymity, diagnosis seems to be more relevant to dropout rates. So, dropout rates found in the studies addressing exclusively participants who showed (sub-threshold) binge eating behaviour $[12,13]$ were relatively low, at $24.3 \%$ and $9 \%$. These dropout rates are in accordance with studies of noninternet-based self-help techniques for those patients [63]. It is hypothesized that patients suffering from binge eating are highly motivated to work on their eating problems due to the association of binge eating with high psychological impairment and related health problems $[64,65]$. Apart from anonymity and diagnosis, it is worth noting that dropout rates for internet-based interventions in other psychiatric disorders generally differ widely. For instance, Titov and colleagues [66] found a dropout rate of $11 \%$ in a trial of clinician-assisted internetdelivered CBT for depression, while Spek et al. [31] reported a dropout rate of $66 \%$ for internet-delivered CBT for sub-threshold depression.

In the literature, several different factors (e.g. duration of treatment and amount of contact) have been found to be associated with differences in treatment effects. For example, the influence of therapist support on treatment outcomes for depression was found to be strong $[26,27]$. Furthermore, guided self-help has been shown to produce larger treatment effects than pure self-help in BED $[53,67]$ and providing guidance might increase both adherence to and the benefits of computerized interventions [68]. Unfortunately, it was not possible to draw conclusions in this review about how much influence the duration of an internet-based intervention or the amount of contact with the coaches has on its efficacy, since variability of these two factors was limited between studies. When combined with the different kinds of treatments and participants addressed, no clear conclusion can be drawn.

Although the internet-based interventions discussed in this review were not specifically aimed at reducing comorbid symptoms such as depression, anxiety or at increasing quality of life, these can be assumed to be relevant factors for eating disorder patients. Overall results of the present review indicate that depressive symptoms and anxiety can be reduced by internet-based interventions. This is in line with previous data from face-to-face studies [52]. For example in their meta-analysis, Vocks and colleagues found significant but small mean effect sizes for depression after psychotherapy and structured self-help when comparing experimental and control groups. It can be assumed that improvement of eating disorder symptoms might influence depressiveness and symptoms of anxiety. Furthermore, significant treatment effects found in this review indicate that quality of life and satisfaction with life are related to a reduction of eating disorder symptoms.

\section{Limitations}

Several limitations of this review have to be addressed. For example, only a limited number of studies could be included. The fact that these studies used different kinds of interventions and addressed different eating disorders complicated the process of reaching reliable conclusions. To include more articles, eligibility criteria would have had to be broader, but increasing the variability of the analysed treatment programs, for instance by including prevention programs, would have lead to even less comparability of results. One recommendation would be to carry out the literature search in more databases to assure the inclusion of all relevant articles. But as internetbased interventions for eating disorders are a relatively novel treatment option still undergoing research, we suspect that the electronic search and the subsequent screening of the reference lists described above should lead to the inclusion of all articles relevant for the review.

Finally, the absence of a CBT-based program, the short duration of the intervention and the lack of posttreatment assessment made the study conducted by Johnston et al. [15] difficult to compare with the other studies in the review. Nonetheless, this study fulfilled all selection criteria and therefore needed to be included in this review. Furthermore, although effect sizes of internet-based interventions can be considered medium to high, comparing them to face-to-face interventions will always be difficult, especially with regard to various sample characteristics (e.g. inpatient vs. outpatient). Generally, further research should evaluate the characteristics of samples recruited for internet-based interventions for eating disorders. 


\section{Conclusions}

In summary, internet-based interventions based upon CBT principles can be assumed to be a good alternative to face-to-face therapies for the treatment of eating disorders. Especially internet-based guided self-help programs and self-help books supported by e-mail contact showed promising results. This conclusion is supported by the fact that effect sizes of these interventions are comparable to face-to-face treatments for eating disorders and the apparent stability of the treatment outcomes. Furthermore, treatments delivered via the internet are especially relevant for patients who do not have access to conventional therapy programs due to different reasons.

Unfortunately, due to the small number of studies, the differences in disorders addressed and assessment methods used in each study, these conclusions must be interpreted as promising but not definitive. Further research investigating different treatments and self-help programs is needed to analyze the different components of CBT and to identify the most effective strategies. Additionally, predictors of treatment outcome should be identified and examined in order to better deduce which treatment program fits best for each patient.

In conclusion, despite promising effects which internetbased interventions utilizing structured self-help based upon CBT principles seem to have on patients suffering from different kinds of eating disorders, further research is needed to identify factors that lead to these positive results.

\section{Abbreviations \\ $\mathrm{RCT}$ : Randomized controlled trial; CT: Controlled trial; CBT: Cognitive behavioural therapy; BN: Bulimia nervosa; BED: Binge eating disorder; EDNOS: Eating disorder not other specified; EDI: Eating Disorder Inventory; EDE-Q: Eating Disorder Examination Questionnaire; BITE: Bulimia Investigatory Test Edinburgh; BDI: Beck Depression Inventory; HADS: Hospital Anxiety and Depression Scale; MADRS: MontgomeryÅsberg Depression Scale Self- assessment (MADRS); SWLS: Satisfaction with Life Scale; IWQOL-Lite: Impact of Weight on Quality of Life; WHOQOL-Bref: WHO Quality of Life Questionnaire (short form).}

\section{Competing interests}

The authors declare that they have no competing interests.

\section{Authors' contributions}

Manuscript concept and design: RD, AK, BW. Acquisition of data: RD, AT. Analysis and interpretation of data: RD, BW. Drafting of manuscript: RD, AT, AK, BW. Revision of manuscript: RD, AT, AK, BW. All authors read and approved.

\section{Acknowledgements}

This work was supported by the Federal Ministry of Education and Research (BMBF), Germany, FKZ: $01 \mathrm{EO} 1001$.

Received: 16 August 2012 Accepted: 17 July 2013

Published: 6 August 2013

\section{References}

1. Hoek HW, Van Hoeken D: Review of the prevalence and incidence of eating disorders. Int J Eat Disord 2003, 34(4):383-396.

2. Oliver MI, Pearson N, Coe N, Gunnell D: Help-seeking behaviour in men and women with common mental health problems: cross-sectional study. Br J Psychiatry 2005, 186:297-301.
3. Beintner I, Jacobi C, Taylor CB: Effects of an internet-based prevention programme for eating disorders in the USA and germany-a metaanalytic review. Eur Eat Disord Rev 2012, 20(1):1-8.

4. Bruning Brown J, Winzelberg AJ, Abascal LB, Taylor CB: An evaluation of an internet-delivered eating disorder prevention program for adolescents and their parents. J Adolesc Health 2004, 35(4):290-296.

5. Jacobi C, Morris L, Beckers C, Bronisch-Holtze J, Winter J, Winzelberg AJ, Taylor CB: Maintenance of internet-based prevention: a randomized controlled trial. Int J Eat Disord 2007, 40(2):114-119.

6. Jacobi C, Volker U, Trockel MT, Taylor CB: Effects of an internet-based intervention for subthreshold eating disorders: a randomized controlled trial. Behav Res Ther 2012, 50(2):93-99.

7. Jones M, Luce KH, Osborne MI, Taylor K, Cunning D, Doyle AC, Wilfley DE, Taylor CB: Randomized, controlled trial of an internet-facilitated intervention for reducing binge eating and overweight in adolescents. Pediatrics 2008, 121(3):453-462.

8. Stice $E$, Rohde $P$, Durant $S$, Shaw $H$ : A preliminary trial of a prototype internet dissonance-based eating disorder prevention program for young women with body image concerns. J Consult Clin Psychol 2012, 80(5):907-916.

9. Taylor CB, Bryson S, Luce KH, Cunning D, Doyle AC, Abascal LB, Rockwell R, Dev P, Winzelberg AJ, Wilfley DE: Prevention of eating disorders in at-risk college-age women. Arch Gen Psychiatry 2006, 63(8):881-888.

10. Volker U, Jacobi C, Barr Taylor C: Adaptation and evaluation of an Internet-based prevention program for eating disorders in a sample of women with subclinical eating disorder symptoms: a pilot study. Eat Weight Disord 2011, 16(4):e270-e273.

11. Zabinski MF, Wilfley DE, Calfas KJ, Winzelberg AJ, Taylor CB: An interactive psychoeducational intervention for women at risk of developing an eating disorder. J Consult Clin Psychol 2004, 72(5):914-919.

12. Carrard I, Crepin C, Rouget P, Lam T, Golay A, Van der Linden M: Randomised controlled trial of a guided self-help treatment on the internet for binge eating disorder. Behav Res Ther 2011, 49(8):482-491.

13. Carrard I, Crepin C, Rouget P, Lam T, Van der Linden M, Golay A: Acceptance and efficacy of a guided internet self-help treatment program for obese patients with binge eating disorder. Clin Pract Epidemiol Ment Health 2011, 7:8-18.

14. Fernández-Aranda F, Núñez A, Martínez C, Krug I, Cappozzo M, Carrard I, Rouget $P$, Jiménez-Murcia S, Granero R, Penelo E, et al: Internet-based cognitive-behavioral therapy for bulimia nervosa: a controlled study. Cyberpsychol Behav 2009, 12(1):37-41.

15. Johnston O, Startup H, Lavender A, Godfrey E, Schmidt U: Therapeutic writing as an intervention for symptoms of bulimia nervosa: effects and mechanism of change. Int J Eat Disord 2010, 43(5):405-419.

16. Ljotsson B, Lundin C, Mitsell K, Carlbring P, Ramklint M, Ghaderi A: Remote treatment of bulimia nervosa and binge eating disorder: a randomized trial of Internet-assisted cognitive behavioural therapy. Behav Res Ther 2007, 45(4):649-661.

17. Robinson P, Serfaty M: Getting better byte by byte: a pilot randomised controlled trial of email therapy for bulimia nervosa and binge eating disorder. Eur Eat Disord Rev 2008, 16(2):84-93.

18. Ruwaard J, Lange A, Broeksteeg J, Renteria-Agirre A, Schrieken B, Dolan CV Emmelkamp P: Online cognitive-behavioural treatment of bulimic symptoms: a randomized controlled trial. Clin Psychol Psychother 2012, 20(4):308-318

19. Sanchez-Ortiz VC, Munro C, Stahl D, House J, Startup H, Treasure J, Williams C, Schmidt U: A randomized controlled trial of internet-based cognitivebehavioural therapy for bulimia nervosa or related disorders in a student population. Psychol Med 2011, 41(2):407-417.

20. Heinicke BE, Paxton SJ, MCLean SA, Wertheim EH: Internet-delivered targeted group intervention for body dissatisfaction and disordered eating in adolescent girls: a randomized controlled trial. $J$ Abnorm Child Psychol 2007, 35(3):379-391.

21. Cousineau TM, Franko DL, Trant M, Rancourt D, Ainscough J, Chaudhuri A, Brevard J: Teaching adolescents about changing bodies: randomized controlled trial of an internet puberty education and body dissatisfaction prevention program. Body Image 2010, 7(4):296-300

22. Arcelus J, Mitchell AJ, Wales J, Nielsen S: Mortality rates in patients with anorexia nervosa and other eating disorders. A meta-analysis of 36 studies. Arch Gen Psychiatry 2011, 68(7):724-731.

23. Kersting A, Schlicht S, Kroker K: Internet therapy. Opportunities and boundaries. Nervenarzt 2009, 80(7):797-804. 
24. Squires DD, Hester RK: Development of a computer-based, brief intervention for drinkers: the increasing role of computers in the assessment and treatment of addictive behaviors. the Behavior Therapist 2002, 25(3):59-65.

25. Bosworth K, Espelage D, DuBay T: A computer-based violence prevention intervention for young adolescents: pilot study. Adolescence 1998, 33(132):785-795

26. Andersson G, Cuijpers P: Internet-based and other computerized psychological treatments for adult depression: a meta-analysis. Cogn Behav Ther 2009, 38(4):196-205.

27. Johansson R, Andersson G: Internet-based psychological treatments for depression. Expert Rev Neurother 2012, 12(7):861-869. quiz 870.

28. Calear AL, Christensen $\mathrm{H}$ : Review of internet-based prevention and treatment programs for anxiety and depression in children and adolescents. Med J Aust 2010, 192(11 Suppl):S12-S14

29. Griffiths KM, Farrer L, Christensen $H$ : The efficacy of internet interventions for depression and anxiety disorders: a review of randomised controlled trials. Med J Aust 2010, 192(11 Suppl):S4-S11.

30. Mayo-Wilson E: Internet-based cognitive behaviour therapy for symptoms of depression and anxiety: a meta-analysis. Psychol Med 2007 37(8):1211-1212. 1211; author reply.

31. Spek V, Cuijpers P, Nyklicek I, Riper H, Keyzer J, Pop V: Internet-based cognitive behaviour therapy for symptoms of depression and anxiety: a meta-analysis. Psychol Med 2007, 37(3):319-328.

32. Manzoni GM, Pagnini F, Corti S, Molinari E, Castelnuovo G: Internet-based behavioral interventions for obesity: an updated systematic review. Clin Pract Epidemiol Ment Health 2011, 7:19-28.

33. Van den Berg MH, Schoones JW, Vliet Vlieland TP: Internet-based physical activity interventions: a systematic review of the literature. J Med Internet Res 2007, 9(3):e26.

34. Fairburn CG, Beglin SJ: Assessment of eating disorders - interview or self-report questionnaire. Int J Eat Disord 1994, 16(4):363-370.

35. Garner DM, Olmstead MP, Polivy J: Development and validation of a multidimensional eating disorder inventory for anorexia-nervosa and bulimia. Int J Eat Disord 1983, 2(2):15-34.

36. Garner DM: Eating disorder inventory-2 manual. Odessa, FL: Psychological Assessment Resources; 1991.

37. Nevonen L, Broberg AG: Validating the Eating Disorder Inventory-2 (EDI-2) in Sweden. Eat Weight Disord 2001, 6(2):59-67.

38. Norring C, Sohlberg S: Eating disorder inventory in sweden - description, cross-cultural-comparison, and clinical utility. Acta Psychiatr Scand 1988, 78(5):567-575.

39. Henderson M, Freeman CPL: A self-rating scale for bulimia: "the BITE". Br J Psychiatry 1987, 150:18-24.

40. Hedges LV, Olkin I: Statistical methods for meta-analysis. Orlando FL: Academic Press; 1985.

41. Cohen J: Statistical power analysis for the behavioral sciences. Hillsdale, NJ: L Erlbaum Associates; 1988.

42. Beck AT, Ward CH, Mendelson M, Mock J, Erbaugh J: An inventory for measuring depression. Arch Gen Psychiatry 1961, 4:561-571.

43. Beck AT, Steer RA, Brown GK: BDI II, inventaire de dépression de Beck. 2nd edition. Paris: Editions du Centre de Psychologie Appliquée; 1996.

44. Zigmond AS, Snaith RP: The hospital anxiety and depression scale. Acta Psychiatr Scand 1983, 67(6):361-370.

45. Snaith RP, Zigmond AS: Hospital anxiety and depression scale. Windsor: NFER Nelson; 1994

46. Svanborg P, Asberg M: A new self-rating scale for depression and anxiety states based on the comprehensive psychopathological rating scale. Acta Psychiatr Scand 1994, 89(1):21-28.

47. Kolotkin RL, Crosby RD, Kosloski KD, Williams GR: Development of a brief measure to assess quality of life in obesity. Obes Res 2001, 9(2):102-111.

48. Diener E, Emmons RA, Larsen RJ, Griffin S: The satisfaction with life scale. J Pers Assess 1985, 49(1):71-75.

49. Group TW: Development of the World Health Organization WHOQOLBREF quality of life assessment. The WHOQOL Group. Psychol Med 1998, 28(3):551-558

50. Pennebaker JW: Writing about emotional experiences as a therapeutic process. Psychol Sci 1997, 8(3):162-166.

51. Wilson GT, Zandberg LJ: Cognitive-behavioral guided self-help for eating disorders: effectiveness and scalability. Clin Psychol Rev 2012, 32(4):343-357
52. Vocks S, Tuschen-Caffier B, Pietrowsky R, Rustenbach SJ, Kersting A, Herpertz $S$ : Meta-analysis of the effectiveness of psychological and pharmacological treatments for binge eating disorder. Int J Eat Disord 2010, 43(3):205-217.

53. Loeb KL, Wilson GT, Gilbert JS, Labouvie E: Guided and unguided self-help for binge eating. Behav Res Ther 2000, 38(3):259-272.

54. Fairburn CG, Cooper Z, Doll HA, Norman P, O'Connor M: The natural course of bulimia nervosa and binge eating disorder in young women. Arch Gen Psychiatry 2000, 57(7):659-665.

55. Lewandowski LM, Gebing TA, Anthony JL, O'Brien WH: Meta-analysis of cognitive-behavioral treatment studies for bulimia. Clin Psychol Rev 1997 17(7):703-718

56. Garner DM: Psychotherapy outcome research with bulimia nervosa. Psychother Psychosom 1987, 48(1-4):129-140

57. Griffiths RA: Characteristics of dropouts and completers from hypnobehavioral treatment for bulimia nervosa. Int J Eat Disord 1990, 9(2):217-219.

58. Coker S, Vize C, Wade T, Cooper PJ: Patients with bulimia nervosa who fail to engage in cognitive behavior therapy. Int J Eat Disord 1993, 13(1):35-40.

59. Blouin J, Schnarre K, Carter J, Blouin A, Tener L, Zuro C, Barlow J: Factors affecting dropout rate from cognitive-behavioral group treatment for bulimia nervosa. Int J Eat Disord 1995, 17(4):323-329.

60. McKisack C, Waller G: Why is attendance variable at groups for women with bulimia nervosa? The role of eating psychopathology and other characteristics. Int J Eat Disord 1996, 20(2):205-209.

61. Waller G: Drop-out and failure to engage in individual outpatient cognitive behavior therapy for bulimic disorders. Int J Eat Disord 1997, 22(1):35-41.

62. Christensen $\mathrm{H}$, Griffiths KM, Farrer L: Adherence in internet interventions for anxiety and depression: systematic review. J Med Internet Res 2009, 11(2):e13.

63. Grilo CM, Masheb RM: A randomized controlled comparison of guided self-help cognitive behavioral therapy and behavioral weight loss for binge eating disorder. Behav Res Ther 2005, 43(11):1509-1525.

64. Glasofer DR, Tanofsky-Kraff M, Eddy KT, Yanovski SZ, Theim KR, Mirch MC, Ghorbani S, Ranzenhofer LM, Haaga D, Yanovski JA: Binge eating in overweight treatment-seeking adolescents. J Pediatr Psychol 2007, 32(1):95-105.

65. Grucza RA, Przybeck TR, Cloninger CR: Prevalence and correlates of binge eating disorder in a community sample. Compr Psychiatry 2007, 48(2):124-131.

66. Titov N, Andrews G, Davies M, Mclntyre K, Robinson E, Solley K: Internet treatment for depression: a randomized controlled trial comparing clinician vs. technician assistance. PLoS One 2010, 5(6):e10939.

67. Carter JC, Fairburn CG: Cognitive-behavioral self-help for binge eating disorder: a controlled effectiveness study. J Consult Clin Psychol 1998, 66(4):616-623

68. Schmidt U, Andiappan M, Grover M, Robinson S, Perkins S, Dugmore O, Landau S, Treasure J, Eisler I, Williams C: Randomised controlled trial of CD-ROM-based cognitive-behavioural self-care for bulimia nervosa. Br J Psychiatry 2008, 193(6):493-500.

\section{doi:10.1186/1471-244X-13-207}

Cite this article as: Dölemeyer et al:: Internet-based interventions for eating disorders in adults: a systematic review. BMC Psychiatry 2013 13:207

\section{Submit your next manuscript to BioMed Central and take full advantage of:}

- Convenient online submission

- Thorough peer review

- No space constraints or color figure charges

- Immediate publication on acceptance

- Inclusion in PubMed, CAS, Scopus and Google Scholar

- Research which is freely available for redistribution 